\title{
EVALUACIÓN DE LA CALIDAD DE REVISTAS CIENTÍFICAS ESPAÑOLAS: ANÁLISIS DE SUS PROCESOS DE REVISIÓN
}

\author{
María-Ángeles Coslado, Izaskun Lacunza y Germán Ros
}
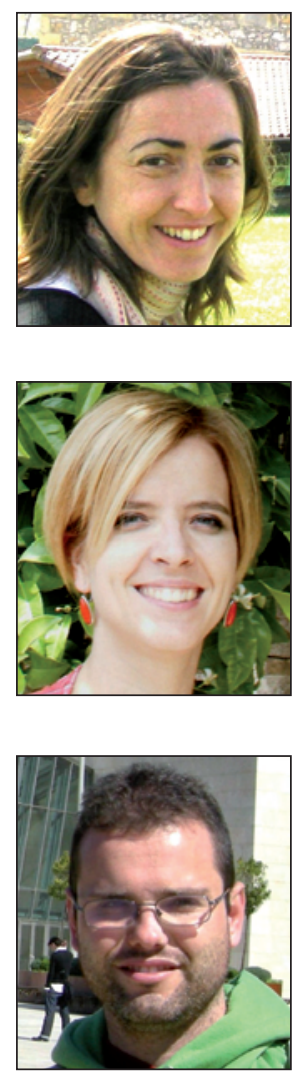

María-Ángeles Coslado es licenciada en filología hispánica (2000) y en documentación (2003) por la Universidad de Alcalá y máster en investigación en documentación por la Universidad Carlos III de Madrid (2009). Trabaja en el Departamento de Gestión de la Información Científica Integrada de la Fundación Española para la Ciencia y la Tecnología (Fecyt), dedicada a la evaluación de revistas científicas y al estudio de indicadores bibliométricos.

Fundación Española para la Ciencia y la Tecnología (Fecyt) Depto. de Gestión de la Información Científica Integrada C/ Pedro Teixeira, 8, planta 2. 28020 Madrid mangeles.coslado@fecyt.es

Izaskun Lacunza es doctora en químicas por la Universidad Autónoma de Madrid en 2006. Es responsable de la unidad de promoción de la producción científica y el acceso abierto de la Fundación Española para la Ciencia y la Tecnología (Fecyt). La unidad se dedica a coordinar las políticas de acceso abierto de carácter regional, nacional y europeo y a promocionar la profesionalización e internacionalización de las revistas científicas españolas.

Fundación Española para la Ciencia y la Tecnología (Fecyt) Depto. de Gestión de la Información Científica Integrada C/ Pedro Teixeira, 8, planta 2. 28020 Madrid izaskun.lacunza@fecyt.es

Germán Ros es doctor en física por la Universidad de Alcalá de Henares en 2009, donde ahora ocupa el puesto de profesor ayudante doctor. Sus principales líneas de investigación son los rayos cósmicos de ultra-alta energía y la materia oscura. Ha participado en más de 20 artículos en revistas internacionales y es fundador de las Jornadas de Jóvenes Investigadores de la Universidad de Alcalá. Recientemente ha publicado algunos trabajos sobre bibliometría.

Universidad de Alcalá Departamento de Física. Edificio de Ciencias 28871 Alcalá de Henares, Madrid german.ros@uah.es

\section{Resumen}

Un deber básico de toda revista científica es la evaluación de los trabajos que publica. La práctica más habitual es la evaluación por pares, en la que varios expertos determinan la validez de las ideas y los resultados, así como el impacto potencial para la ciencia. A partir de la documentación proporcionada por los editores de revistas en la I Edición de la Evaluación de la Calidad de Revistas Científicas Españolas realizada por Fecyt en 2008, se han analizado algunas de las prácticas editoriales: cumplimiento de la evaluación externa, envío de instrucciones a los revisores, existencia de protocolos para evaluar los artículos y anonimato de los agentes implicados en la revisión. Todo el proceso de revisión por pares ha sido contrastado más allá de la declaración de la misma en la publicación. Se ha comprobado que existe una escasa normalización en estos procesos, lo que refleja una falta de profesionalización de las revistas científicas españolas.

\section{Palabras clave}

Arbitraje científico, Revisión por pares, Protocolos de evaluación, Anonimato, Revistas científicas, Revisores, España, Fecyt.

Title: Quality assessment of Spanish scientific journals: analysis of their review processes

\begin{abstract}
One of the basic duties of scientific journals is the evaluation of the papers that they publish. The most common practice is peer review, in which several experts determine the reliability of the ideas and results as well as the potential impact on science. Studying the documentation provided by the editors of Quality Assessment of Spanish Scientific Journals, 1st Edition, in 2008, we have analyzed some of the editorial practices in the evaluation process, such as external evaluation, instructions to referees, the existence of protocols to evaluate the articles and anonymity of those involved in the review. The
\end{abstract}


significance of our work is that the evaluation process has been checked independently of what is stated in the journal. We have verified that there is a lack of standardization in the evaluation process, which is symptomatic of the need for deeper professionalization.

\section{Keywords}

Evaluation, Peer review, Protocols to evaluate, Anonymity, Scientific journals, Referees, Spain, Fecyt.

Coslado, María-Ángeles; Lacunza, Izaskun; Ros, Germán. "Evaluación de la calidad de revistas científicas españolas: análisis de sus procesos de revisión”. El profesional de la información, 2011, marzo-abril, v. 20, n. 2, pp. 159-164.

DOI: 10.3145/epi.2011.mar.05

\section{Introducción}

La revisión por pares en las publicaciones científicas es aquella en la que dos o más evaluadores con un nivel de conocimientos similar al del autor leen y analizan los artículos para determinar la validez de las ideas y los resultados, y cuál es el impacto potencial de ese trabajo en el mundo de la ciencia (Campanario, 2002). Laudel (2006) emplea estas palabras: práctica en la cual un trabajo de investigación es evaluado por expertos que trabajan en el mismo campo sobre temas similares. Se ha demostrado que es el método más apropiado para valorar el grado de desarrollo de un determinado campo de investigación (Bordons; Zulueta, 1999).

El sistema de revisión por pares de una revista científica está concebido como una ayuda al editor. Por un lado los revisores juzgan la consistencia científica del trabajo y la originalidad y finalmente los editores toman la decisión final de aceptar o rechazar un determinado trabajo. A veces el arbitraje científico se encarga también de garantizar que los trabajos presentados cumplan las normas de publicación y edición científica de la revista (Garfield, 1986), aunque en general de los aspectos más formales se encarga el personal de la Redacción.

En esta línea se han publicado numerosos trabajos. Destaca el libro de Hames Peer review and manuscripts management in scientific journals: guidelines for good practice (Hames, 2007) en el que se explican de forma detallada los procesos de evaluación de los trabajos así como la forma de llevar a cabo una adecuada gestión de los evaluadores. También se puede consultar el trabajo Guidelines on good refereeing practice, en el manual Science editors' handbook publicado por la European Association of Science Editors (Enckell, 1999).

Los procedimientos de arbitraje científico varían dependiendo del tipo de manuscrito, de revista y del campo al que pertenezca. En la revisión por pares se suelen utilizar dos evaluadores por artículo. Estos no siempre coinciden en sus evaluaciones y cuando hay grandes diferencias los editores pueden enviar el manuscrito a otros adjuntando o no las observaciones de los primeros (Garfield, 1986).

La elección de revisores es una de las funciones de los editores de las revistas. Un buen editor debe tener una buena cantera de evaluadores que representen las distintas especialidades que trata su revista y que hayan aceptado colaborar. Los editores tienen que estar al corriente de quiénes son los expertos más cualificados para evaluar un trabajo determinado. Hay revistas que poseen una base de datos de revisores, que no suele ser pública (Campanario, 1998), aunque es costumbre que las revistas publiquen periódicamente (por ej., cada 2 años) una lista de las personas que han actuado de revisores. En algunas ocasiones se sugiere a los mismos autores que proporcionen a la revista nombres de posibles revisores dentro de la temática de su artículo.

Junto con el manuscrito los evaluadores suelen recibir una lista de instrucciones y un formulario para poner los comentarios y las recomendaciones al trabajo en cuestión. Éstos son los que se llamarán de ahora en adelante "protocolos de evaluación". Habitualmente los revisores tienen que responder a las pocas semanas expresando su recomendación: el rechazo, la aceptación o solicitando modificaciones en el documento. A menudo incluyen comentarios específicos tanto para el autor como para el editor (Garfield, 1986).

En el contexto internacional, en la evaluación que realiza Thomson Reuters para seleccionar revistas para la Web of Science (WoS), la revisión por pares es un indicador que pertenece al cumplimiento de los estándares mínimos de publicación y que evidencia la calidad de los trabajos publicados. Para analizar este indicador se tienen en cuenta la declaración en las normas para autores sobre el proceso de evaluación y revisión de originales, y la transparencia informativa sobre dicho proceso (Ruiz-Pérez; Delgado-LópezCózar; Jiménez-Contreras, 2006).

En España numerosos trabajos analizan las prácticas editoriales de las publicaciones. Alcaín-Partearroyo y Román-Román (2005) en un estudio sobre revistas de psicología afirman que la existencia de evaluadores externos al consejo de redacción o a la entidad editora de la revista es un elemento de calidad de gran importancia. En esta misma línea, Giménez-Toledo, Román-Román y Sánchez-Nistal (1999) estudian en revistas españolas de economía si se realiza una revisión externa de los trabajos. En ambos se comprueba el cumplimiento de este indicador en la declaración en la propia revista. Por su parte Delgado-López-Cózar (2001) analiza las deficiencias que presentan las revistas de documentación, y destaca la poca profesionalización de las publicaciones y la poca rigurosidad que presentan en la evaluación de los manuscritos.

De todo ello se deduce la necesidad de un análisis global de las prácticas reales de los procesos de evaluación en las revistas científicas españolas. 
El objetivo principal de este trabajo es estudiar algunas de las prácticas editoriales en las revistas participantes en la I edición de la Evaluación de la calidad de las revistas científicas españolas llevada a cabo por la Fundación Española para la Ciencia y la Tecnología (Fecyt) en 2008. Un análisis más detallado de la metodología y los resultados de este proceso se puede consultar en Coslado, Báez y Lacunza, 2010 y en Coslado, 2009.

El proceso de evaluación de Fecyt tiene como precedentes el proyecto europeo European reference index for the humanities (ERIC), la plataforma Difusión y calidad editorial de las revistas españolas de humanidades y ciencias sociales y jurídicas (DICE), Latindex o el que realiza Thomson Reuters, entre otros. Todos ellos consideran los sistemas de arbitraje como parte importante en sus evaluaciones. La originalidad del trabajo aquí presentado consiste en que, a diferencia de los procesos precedentes, se ha contrastado mediante la solicitud de documentación a los editores la realización de todo el proceso de la revisión por pares externa de los trabajos más allá de la declaración de la misma en la publicación.

\section{Metodología}

La muestra para este trabajo ha sido tomada de las revistas evaluadas en la I edición de la Evaluación de la calidad de las revistas científicas españolas (Fecyt) y está constituida por 119 revistas, de las que se han analizado varios parámetros relacionados con el arbitraje científico, que se describen en esta sección.

Para valorar el tipo de arbitraje científico que realizaban a la hora de seleccionar los manuscritos, se solicitó a las revistas que enviaran los informes de evaluación de dos trabajos elegidos al azar por Fecyt, así como las comunicaciones que se habían mantenido entre los agentes implicados en el proceso de revisión (autor, editor y revisor). Para verificar que éste es externo se solicitó que enviaran una lista con los revisores (nombre y filiaciones profesionales) que habían participado en el proceso editorial durante el año 2007.

Dentro de las prácticas editoriales de revisión por pares de esta muestra, se analizaron los siguientes parámetros:

- si la revisión por pares es externa;

- existencia de protocolos para evaluar los artículos;

- si proporcionan instrucciones a los revisores;

- anonimato de los agentes implicados en la revisión.

Existe cierta confusión en la denominación de los dos consejos (de redacción y científico) que debe tener una revista

\section{Arbitraje científico externo}

Se ha contrastado con la lista de revisores de 2007 que enviaron los editores. Se ha considerado que no cumplen con este parámetro aquellas revistas en las que el 50\% o más de los revisores pertenecen al consejo de redacción o a la entidad editora de la revista.
Existe cierta confusión en la denominación de los dos consejos (de redacción y científico) que debe tener una revista, hecho que se ha observado también en las que se han evaluado en este proceso. Se ha considerado que, más allá de la denominación dada por la propia publicación, el consejo de redacción es aquel cuyas funciones están relacionadas con el proceso de edición y política editorial de la revista: el encargado de preparar los números, la aceptación de los originales y de establecer relaciones con los autores, los evaluadores y el consejo científico o asesor. Éste último, formado por científicos altamente especializados y de prestigio en el área de la revista, tiene como funciones principales la representación, la promoción internacional y la evaluación de los trabajos (Román-Román, 2008).

\section{Protocolos para evaluar los artículos}

En las Jornadas de difusión de los resultados de la I Evaluación de la calidad de las revistas científicas españolas, que organizó Fecyt en Madrid en junio de 2008, se expuso una serie de funciones que deberían cumplir los protocolos de evaluación que servían de guía a los revisores. Una era la transparencia editorial. Los protocolos aseguran que tanto los revisores como los autores conozcan los criterios que se emplean en la evaluación de los manuscritos (Ruiz-Pérez, 2008). Incluyen instrucciones sobre los siguientes aspectos: 1) técnicos-formales, 2) de contenido, 3) mejora de la presentación formal y los contenidos científicos, 4) cómo se debe formular la decisión final, y 5) recomendación sobre la prioridad en la publicación (Ruiz-Pérez, 2008).

A partir de los informes solicitados de evaluación de los artículos, se analizó en primer lugar si los protocolos cumplían satisfactoriamente los aspectos descritos anteriormente. En los casos en que se consideron válidos se estudió por un lado su idioma con el fin de ver la internacionalización de la cantera de revisores de la revista, y por otro, si se indicaba al revisor que valorara explícitamente la originalidad del manuscrito.

\section{Instrucciones a los revisores}

Los protocolos facilitan al editor la decisión final de publicar un trabajo (Ruiz-Pérez, 2008).

En esta línea se ha analizado si los editores envían las pautas sobre varios aspectos que deben seguir los revisores:

- disponibilidad o capacidad de llevar a cabo esta tarea;

- utilización del protocolo como guía;

- establecimiento de un plazo de tiempo;

- existencia de algún conflicto de intereses;

- mantenimiento de la confidencialidad en cuanto al manuscrito y a la revisión;

- compensación económica o de reconocimiento académico por parte de la revista.

\section{Anonimato de los agentes implicados en la revisión}

Una de las características principales de la revisión por pares de los artículos es mantener ocultas las identidades de los revisores y de los autores en el proceso de evaluación de los trabajos. Se ha analizado el tipo de anonimato de los agentes implicados en la revisión, distinguiéndose entre:

- doble ciego: se oculta la identidad del autor al revisor y viceversa. 
- simple: se oculta al autor la identidad del revisor.

- se oculta la identidad del autor, mientras el revisor opta por mantener su anonimato. Es decir, el editor le pregunta al revisor si desea o no permanecer en el anonimato.

Para estudiar este parámetro se han tenido en cuenta las comunicaciones mantenidas por los agentes y los informes de evaluación de los dos artículos elegidos al azar, y con ello se han clasificado en uno de los tipos anteriores.

Aunque la mayoría de revistas realiza revisión por pares, existe una falta de normalización en los protocolos de evaluación

\section{Resultados}

En primer lugar se ha verificado la existencia de arbitraje científico externo y se ha comprobado que 32 de 119 revistas $(27 \%)$ no cumplían con este criterio.

De las 87 revistas que sí superaron el criterio, 16 no presentan protocolos que cumplan las funciones básicas de los mismos, por lo que fueron descartadas del subsiguiente análisis.

Se han analizado dos aspectos de los protocolos: el idioma en el que se presentan y si se pide de manera explícita que se valore la originalidad de los manuscritos. Los resultados muestran que predominan las revistas que sólo presentan los protocolos en castellano (72\%), como se puede observar en el gráfico 1 , y que se pide explícitamente al revisor que valore la originalidad del trabajo en el $69 \%$ de los casos.

En cuanto a las instrucciones a los revisores, un $72 \%$ de las revistas da detalles de cómo tienen que llevar a cabo la revisión, atendiendo en todo caso a la política editorial de la revista. En los casos que presentan instrucciones se observa que:

- Un 57\% les preguntan si están capacitados y tienen disponibilidad para revisar el trabajo que se les envía. En este punto hay que destacar que el $12 \%$ de los que sí realizan esta pregunta piden al revisor que si no puede llevar a cabo la evaluación indique el nombre de otro revisor.

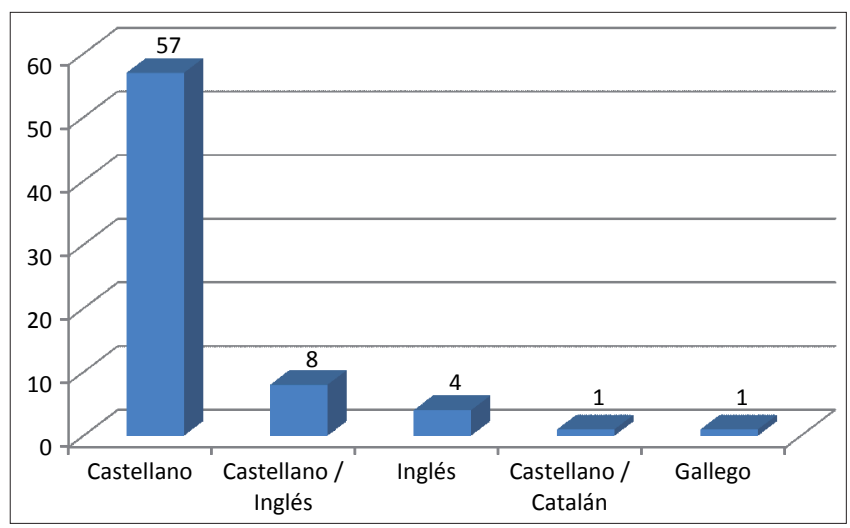

Gráfico 1. Idioma de los protocolos de evaluación
- El 69\% indica al evaluador que debe seguir como guía el protocolo que se le envía.

- El 67\% indica el plazo de tiempo que tiene el revisor para hacer la evaluación. En el gráfico 2 se puede observar que el plazo que más se repite está alrededor de un mes. La categoría "sin especificar" se refiere a aquellas instrucciones que dejan el espacio en blanco para que el editor ponga un plazo determinado teniendo en cuenta la política editorial del momento o las características del manuscrito.

- En el 84\% de las instrucciones para los revisores no se les pregunta si existe algún conflicto de intereses para hacer la revisión.

- Un 94\% no hace alusión al mantenimiento de la confidencialidad del manuscrito y de la revisión.

- El 94\% de las instrucciones no indica que el revisor vaya a percibir una compensación por el trabajo que va a realizar. Del 6\% restante, hay que destacar que sólo 2 revistas ofrecen una compensación económica y una de ellas un reconocimiento académico.

Por último se analizó el anonimato durante el proceso de revisión de los artículos. Mediante las comunicaciones entre los agentes implicados en el proceso y de los informes de evaluación, se pudo comprobar el tipo de anonimato (ver gráfico 3). Se destaca que el $85 \%$ de la documentación analizada presenta doble ciego, es decir que el autor no conoce la identidad del revisor ni viceversa.

\section{Conclusiones}

En este trabajo se han analizado las prácticas editoriales llevadas a cabo en los procesos de revisión de los trabajos de 119 revistas científicas españolas mediante el análisis de la documentación solicitada por los editores y no sólo en la

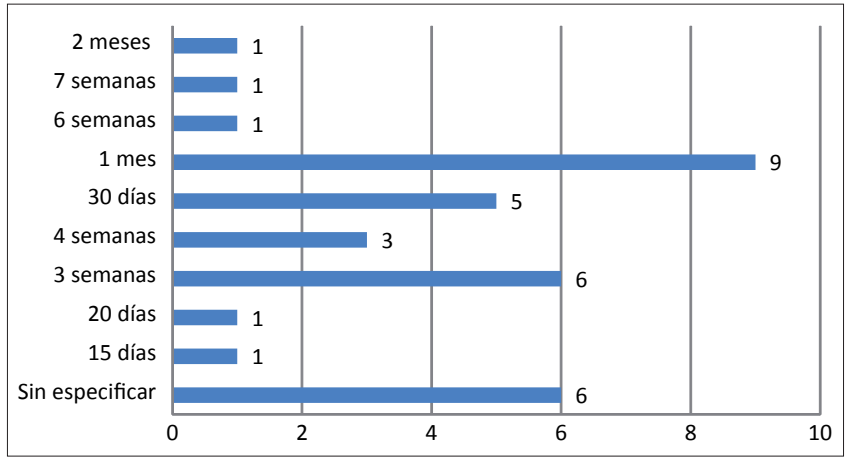

Gráfico 2. Distribución de los plazos de tiempo para la revisión del trabajo

Anonimato en la revisión en la evaluación Fecyt 2008

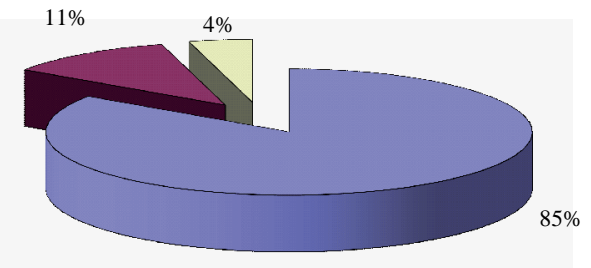

Doble ciego (60)

Anonimato del revisor (8) DOpción del anonimato del revisor (3)

Gráfico 3. Anonimato en el arbitraje científico 
declaración de la propia revista. Es destacable que un $27 \%$ no realiza revisión por pares externa.

Respecto a los protocolos de evaluación estudiados, un 18\% no cumplían las funciones básicas exigibles a los mismos, esto es, asegurar que los revisores conozcan los criterios que se emplean en la revisión y la transparencia editorial. Este porcentaje es importante teniendo en cuenta que aunque estas revistas sí cumplían la revisión externa por pares, no presentaban una normalización del proceso.

En cuanto a los protocolos de evaluación hay que destacar también que:

- El idioma que predomina es el castellano, de hecho un $72 \%$ presenta los protocolos sólo en este idioma. Esto indica que los revisores con los que cuenta la revista son todos de ámbito nacional o se limita a expertos hispano hablantes, y por tanto refleja una falta de internacionalización, o muy limitada, en la revisión de los trabajos.

- Prácticamente un tercio de los casos no exige explícitamente al revisor que valore la originalidad del trabajo, probablemente dando ésta de manera errónea por supuesta.

Por otro lado, del análisis de las instrucciones enviadas por el editor al revisor cabe mencionar:

- Casi en la totalidad de las revistas no se indica de forma explícita que se mantenga la confidencialidad tanto del manuscrito como del proceso de evaluación del trabajo.

- El trabajo de los revisores es una labor altruista. El 94\% de las instrucciones analizadas no indican que los revisores perciban una compensación económica ni un reconocimiento académico.

Los resultados son sintomáticos de una falta de profesionalización en un amplio espectro de las revistas científicas españolas

Todo ello junto con la falta de homogeneidad en los plazos de las revisiones y en la exigencia de requisitos mínimos a los revisores (si están capacitados y tienen disponibilidad para la revisión, si existe algún conflicto de intereses) muestra que, a pesar de que en la mayoría de los casos analizados se lleva a cabo un proceso de revisión por pares externo, existe una falta de normalización en los protocolos de evaluación utilizados.

La muestra no es suficientemente elevada como para poder extraer conclusiones generales acerca de cuál es el panorama nacional en las prácticas editoriales, pero los resultados expuestos son sintomáticos de una falta de profesionalización en un amplio espectro de las revistas científicas nacionales.

\section{Bibliografía}

Alcaín-Partearroyo, María-Dolores; Román-Román, Adelaida. "Hacia una valoración integrada de las revistas españolas de ciencias sociales y humanas: las revistas de psicología". Psicothema, 2005, v. 17, n. 2, pp. 179-189. http://www.psicothema.com/pdf/3086.pdf
Bordons, María; Zulueta, María-Ángeles. "Evaluación de la actividad científica a través de indicadores bibliométricos". Revista española de cardiología, 1999, v. 52, n. 10. http://www.elsevier.es/cardio/ctl_servlet? f=60\&ident $=190$

Campanario, Juan-Miguel. "Peer review for journals as it stands today - Part 1". Science communication, 1998, v. 19, n. 3, pp. 181-211

http://www2.uah.es/jmc/ai4.pdf

Campanario, Juan-Miguel. "Sistema de revisión por expertos (peer review): muchos problemas y pocas soluciones". Revista española de documentación científica, 2002, v. 25, n. 3, pp. 267-285.

http://redc.revistas.csic.es/index.php/redc/article/viewArti cle/107

Coslado, María-Ángeles. Análisis del sistema de evaluación de revistas científicas españolas desarrollado por la Fundación Española para la Ciencia y la Tecnología en el año 2008, 2009. (Proyecto de tesis - Universidad Carlos III de Madrid).

Coslado, María-Ángeles; Báez, José-Manuel; Lacunza, Izaskun. "Descripción y análisis del proceso de evaluación de la calidad de las revistas científicas españolas llevado a cabo por Fecyt en el año 2008". Revista española de documentación científica, 2010, v. 33, n. 3, pp. 481-495.

http://redc.revistas.csic.es/index.php/redc/article/view/ $567 / 642$

Delgado-López-Cózar, Emilio. “Las revistas españolas de ciencias de la documentación: productos manifiestamente mejorables". El profesional de la información, 2001, v. 10, n. 12, pp. 46-56.

http://www.elprofesionaldelainformacion.com/contenidos/ 2001/diciembre/10.pdf

Enckell, Pehr. "Guidelines on good refereeing practice". Science editors' handbook (C. 1-3.1). European Association of Science Editors, 1999.

Garfield, Eugene. "Refereeing and peer review. Part 1. Opinion and conjecture on the effectiveness of refereeing". Current contents, 1986, n. 31, pp. 3-11.

http://www.garfield.library.upenn.edu/essays/v9p230y 1986.pdf

Giménez-Toledo, Elea; Román-Román, Adelaida; SánchezNistal, José-María. "Aplicación de un modelo de evaluación a las revistas científicas españolas de economía: una aproximación metodológica". Revista española de documentación científica, 1999, v. 23, n. 3, pp. 309-324.

http://redc.revistas.csic.es/index.php/redc/article/down load/339/553

Hames, Irene. Peer review and manuscripts management in scientific journals: guidelines for good practice. Malden [USA]: Blackwell Publishing; Association of Learned and Professional Society Publishers (Alpsp), 2007.

Laudel, Grit. "Conclave in the Tower of Babel: how peers review interdisciplary research proposals". Research evaluation, 2006, v. 15, n. 1, pp. 57-68.

http://www.laudel.info/pdf/journal\%20articles/06\%20con clave\%20in\%20the\%20tower.pdf 
Rip, Arie. "La república de la ciencia en los años noventa". Zona abierta, 1996, 75-76, pp. 57-89.

http://doc.utwente.nl/34144/1/Rip96republica.pdf

Román-Román, Adelaida. "Recomendaciones sobre los siguientes indicadores: periodicidad, consejo de redacción y consejo asesor y evaluación por pares externa". En: Jornadas de difusión de los resultados de la I Evaluación voluntaria de la calidad de las revistas científicas españolas, Madrid, junio 2008.

http://recyt.fecyt.es/documentos/4.ppt

Ruiz-Pérez, Rafael. "Calidad editorial: instrucciones a autores y protocolos". En: Jornadas de difusión de los resultados de la I Evaluación voluntaria de la calidad de las revistas científicas españolas, Madrid, junio 2008. http://recyt.fecyt.es/documentos/5.ppt

Ruiz-Pérez, Rafael; Delgado-López-Cózar, Emilio; JiménezContreras, Evaristo. "Criterios del Institute for Scientific Information para la selección de revistas científicas. Su aplicación a las revistas españolas: metodologías e indicadores". Intl journal of clinical and health psychology, 2006, v. 6, n. 2, pp. 401-424

http://www.aepc.es/ijchp/articulos_pdf/ijchp-184.pdf

Sanz-Menéndez, Luis. "Evaluación de la investigación y sistema de ciencia". Boletín de la Sociedad Española de Bioquímica y Biología Molecular, 2004, 140, pp. 6-10.

http://digital.csic.es/bitstream/10261/1605/1/dt-0407.pdf
IMPLEMENTING

TECHNOLOGY SOLUTIONS IN LIBRARIES

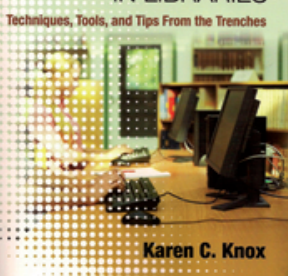

Implementing technology solutions in libraries techniques, tools, and tips from the trenches Karen Knox

El libro "Implementación de soluciones tecnológicas en las bibliotecas. Técnicas, herramientas y consejos desde la trinchera" presenta enfoques sencillos y prácticos para llevar a cabo proyectos de informatización en bibliotecas. Está dirigido a las personas que realizan la introducción de automatización de procesos, cosa que puede consumir una parte sustancial del presupuesto de la biblioteca. Karen Knox, directora de tecnologías de la información, desmenuza la ejecución de un proyecto, desde la planificación hasta la evaluación, examinando cuidadosamente cada paso.

La autora ha puesto en marcha muchos proyectos técnicos en los últimos años, algunos con más éxito que otros, como se apresura a admitir, y basándose en su experiencia ayuda a los lectores a identificar los componentes más críticos de cualquier proyecto, así como a modificarlo y ampliarlo para satisfacer las necesidades específicas de cada biblioteca. El conjunto de consejos, trucos, técnicas y herramientas que Karen comparte aquí dice que aseguran el éxito en la implementación de proyectos bibliotecarios.

Febr. 2011, 192 pp. ISBN 978157387403 8. Precio: \$35,00. Information Today http://www.infotoday.com

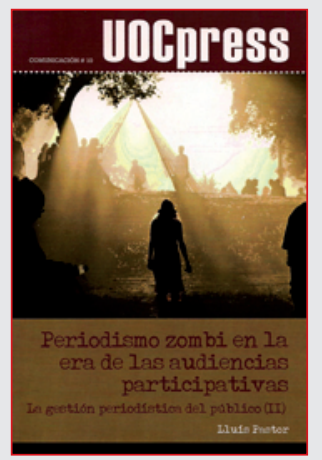

\section{Periodismo zombi en la era de las audiencias participativas. La gestión periodística del público (II) Lluís Pastor}

El periodismo zombi es el periodismo que se ha visto obligado a incorporar la voz del público en los medios de comunicación pero que no sabe qué hacer periodísticamente con eso. Es un periodismo que parece vivo pero que está muerto. Que balbucea, que trastabilla. Que no sabe cómo mejorar el producto periodístico con la integración de las voces de la audiencia. El libro analiza cómo es la participación de los ciudadanos en los diarios digitales europeos y propone algunas vías para convertirla en algo que mejore la oferta periodística que preparan los medios de comunicación.

El periodismo zombi debe saber cambiar, debe saber derrumbar los prejuicios que los periodistas tienen sobre el público y debe atreverse a integrar nuevos procesos y nuevas funciones que conviertan a los ciudadanos que forman la audiencia en colaboradores de los periodistas. En este sentido, la fórmula de este nuevo periodismo cambiante y mutante se basa en tres principios: el de comunidad, el de meta mediación y el de gestión del público. 